\title{
BOUNDS ON FRONT SPEEDS FOR INVISCID AND VISCOUS G-EQUATIONS*
}

\author{
JAMES NOLEN ${ }^{\dagger}$, JACK XIN ${ }^{\ddagger}$, AND YIFENG YU§
}

\begin{abstract}
G-equations are well-known front propagation models in combustion and describe the front motion law in the form of local normal velocity equal to a constant (laminar speed) plus the normal projection of fluid velocity. In level set formulation, G-equations are Hamilton-Jacobi equations with convex ( $L^{1}$ type) but non-coercive Hamiltonians. We study front speeds of both inviscid and viscous G-equations in mean zero flows, and compare the qualitative speed properties with those of quadratically nonlinear Hamilton-Jacobi equations and KPP (Kolmogorov-PetrovskyPiskunov) fronts (with minimal speed). For the inviscid case, we analyze a variational solution formula (control representation) by choosing suitable test functions. For the viscous case, we analyze traveling front equations which agree with the cell problem of homogenization. We found that viscosity can drastically alter the front speed growth law of G-equations. Without viscosity, front speed grows like $O(A / \log A)$ in cellular flows of large amplitude $A$. With proper viscosity, the front speed grows no faster than $O(\sqrt{\log A})$. In contrast, the KPP front speed grows like $O\left(A^{1 / 4}\right)$ in general cellular flows at any fixed viscosity. The $L^{1}$ type nonlinearity appearing in the G-equation makes the key difference.
\end{abstract}

Key words. Non-coercive Hamilton-Jacobi equations, inviscid-viscous fronts, cellular flows, distinct speed growth laws.

AMS subject classifications. 70H20, 76M50, 76M45, 76N20

1. Introduction. Front or interface propagation in fluid flows is a ubiquitous nonlinear phenomenon in various areas of science and technology such as chemical reaction fronts in liquids and premixed flame propagation in fluid turbulence [28, 26]. Mathematical models range from reaction-diffusion-advection equations (RDA) to advective Hamilton-Jacobi equations (HJ), [32, 34]. A particular HJ equation, the so called G-equation, is most popular in the combustion science literature, [19, 30, 35, 5] among others. The $\mathrm{G}$ equation reads:

$$
G_{t}+v(x) \cdot \nabla G=s_{l}|\nabla G|+\kappa \Delta G,
$$

where $G$ is a scalar function, $v(x)$ is a prescribed flow velocity field, $s_{l}$ is a positive constant (laminar front speed), $\kappa \geq 0$ is a diffusion coefficient. If $\kappa=0$ (inviscid regime), the G-equation (1.1) is the level set equation of the interface motion law: the exterior normal velocity of the interface equals the laminar speed $s_{l}$ plus the projection of the fluid velocity along the normal, see chapter 6 of [24] and [25]. The viscous term $\kappa \Delta G$ introduces an additional length scale; $\kappa>0$ is proportional to the so called Markstein length [5, 25]. The viscous term is also proposed as a simplification of curvature [5]. The fundamental problem in turbulent combustion is to study the large time front speed, or the asymptotic growth rate $\lim _{t \rightarrow+\infty} G(x, t) / t$, and analyze its dependence on parameters of the advection $v$. Such a limit (if it exists) is called the turbulent front speed $\left(s_{T}\right),[26,34]$.

\footnotetext{
*Received July 17, 2009; accepted for publication January 20, 2010. edu).

${ }^{\dagger}$ Department of Mathematics, Duke University, Durham, NC 27708, USA (nolen@math.duke.

${ }^{\ddagger}$ Department of Mathematics, University of California at Irvine, Irvine, CA 92697, USA (jxin@ math.uci.edu).

$\S$ Department of Mathematics, University of California at Irvine, Irvine, CA 92697 USA (yyu1@ math.uci.edu).
} 
Another approach is based on reaction-diffusion theory and begins with a coupled system of RDA equations. The system in the case of equal species diffusion (unit Lewis number) reduces to a reactive passive scalar equation $[26,34]$ :

$$
u_{t}+v(x) \cdot \nabla u=\kappa \Delta u+\frac{1}{\tau_{r}} f(u) .
$$

$f(u)$ a nonlinear reaction function, $f(0)=f(1)=0$. The quadratic reaction is $f(u)=u(1-u)$, or more generally $0<f(u)<u f^{\prime}(0)$ for $u \in(0,1)$, the so called Kolmogorov-Petrovsky-Piskunov (KPP) nonlinearity. The two positive constants are: $\kappa$, the species diffusion constant; $\tau_{r}$, the reaction time scale. If the initial data of $u$ is a nonnegative function of compact support, the large time behavior of $u$ is a propagating front. Fronts also form and propagate under other reaction nonlinearities with proper initial data, such as ignition and bistable nonlinearities [32,34]. The question of large time front speed and its dependence on flows is posed similar to that for G-equations. In many classes of flows, qualitative properties of non-KPP front speeds are found to agree with those of KPP speeds ([4, 20, 36, 34, 38] and references therein).

Though the inviscid G-equation can be derived from ignition or bistable RDA equations in hyperbolic scaling limits $\left(\kappa=\tau_{r} \rightarrow 0\right)[3,32,34]$, the large time front speeds can be quite different due to the effects of multiscale structures in the flow field and slow diffusion in longer time scales. A comparison of front speeds in KPP and G-equations for shear flows with non-zero mean [7] showed that the front speeds from G-equation can be much less than the KPP speeds. The amount of discrepancy may vary depending on the alignment of the mean flow and the shear flow.

In this paper, we compare reaction-diffusion front speeds (KPP and non-KPP) and those of inviscid and viscous G-equations for mean zero flows including gradient flows, shear flows, and cellular flows. The large time front speed for the (inviscid) Gequation is same as the homogenized Hamiltonian of G-equation. The Hamiltonian of the G-equation is $H(p, x, t)=s_{l}|p|-v(x) \cdot p$, which is not coercive, or $H \nrightarrow+\infty$ for all $x$ if $|p| \rightarrow \infty$, as long as the amplitude of $v$ is large enough. The existing theory of HJ homogenization does not apply $[8,15,18,16,17,13,27,31]$. However, a variational control formula for the solution allows us to estimate front speeds by selecting trial controls and extracting their dependence on flow structures and scaling laws in the regime of large advection. Divergence of the front speed in unbounded shear flows appears in the G-equation similarly to KPP [33] and quadratically nonlinear HJs [6]. Further qualitative agreement between KPP fronts and G-equation fronts is found in the slow down of front speeds in gradient flow, and linear growth in shear flows. For the G-equation, it is the behavior of optimally controled characteristics that determine the front speed. For KPP fronts, it is drift-diffusion processes (Ito equations). In the above examples, the presence of viscosity in KPP and the different nonlinearities in KPP and G-equations do not matter much. However for cellular flows in two dimensions, inviscid G-equation front speed grows like $O(\delta / \log \delta)$ if $v$ is scaled to $\delta v$, with $\delta \gg 1,[1,29]$. In contrast, the (minimal) front speed of nonnegative reactions (including KPP and ignition) grows like $O\left(\delta^{1 / 4}\right)$ for any viscosity coefficient $\kappa>0$, $[2,22,38]$.

Does the viscous G-equation agree better with KPP (or combustion type) reaction-diffusion models in cellular flows? Surprisingly, the answer is no, if $\kappa$ is sufficiently larger than $s_{l}$, but independent of $v$. The viscosity term in G-equation reduces the speed enhancement dramatically. An upper bound of viscous front speed is $O(\sqrt{\log \delta})$ in the regime $\delta \gg \kappa \gg s_{l}$. A simple way to explain this new phenomenon 
is to notice that the nonlinearity in the G-equation is $L^{1}$ type, or $s_{T}=s_{l} \mathbb{E}[|\nabla G|]$ at large $t$, where $\mathbb{E}[\cdot]$ is the ensemble (cell) average in the random (periodic) flows. On the other hand, KPP fronts are related to viscous HJ with quadratic nonlinearity and the front speed is approximately $s_{T}=s_{l} \mathbb{E}\left[|\nabla u|^{2}\right]$. The $L^{1}$ and $L^{2}$ norms give very different results for cellular flows where the key contribution resides in narrow boundary layers near separatrices at large $\delta$. Our analysis relies on estimates of advection-diffusion equations and properties of cellular flows [10, 12, 22, 23].

The paper is organized as follows. In section 2 , we outline the minimization formula or control representation of inviscid G-equation. In section 3, we show examples and bounds of front speeds of the inviscid G-equation based on the control formula. This approach extends the treatment of [29], and allows one to extract the unique limiting asymptotics or homogenization from a Lagrangian point of view. In section 4 , we derive the standard cell problem of homogenization of the viscous G-equation by looking for a traveling front, then estimate solutions of the cell problem based on asymptotic properties of cellular flows. We present a rigorous bound on slowly growing speeds for the G-equation in large amplitude cellular flows, and give heuristic explanation of the slowly growing speeds using the stochastic version of the minimization formula or control representation of viscous G-equation. In section 5, we conclude with remarks on future work.

The work was partially supported by an NSF Postdoctoral Fellowship (JN), NSF grants DMS-0712881 (JX), DMS-0848378 and DMS-0901460 (YY). We thank A. Fannjiang, A. Novikov, and L. Ryzhik for helpful remarks on cellular flows.

2. Representation formulas for G-equations. In the G-equation model, the front $\Gamma_{t}$ is defined to be the zero-level set $\Gamma_{t}=\left\{\begin{array}{ll}x: & G(x, t)=0\}\end{array}\right.$ of a function $G(x, t)$, and the level sets of $G$ move in the direction $\hat{n}$ with speed $s_{n}=s_{n}(x, t)$ given by

$$
s_{n}=s_{l}+v(x) \cdot \hat{n} .
$$

The constant $s_{l}$ is the laminar flame speed, and $\hat{n}=\hat{n}(x, t)=-\nabla G /|\nabla G|$ is a unit vector normal to the level set at the point $(x, t)$. Therefore, at points where $G$ is smooth and $|\nabla G| \neq 0, G$ satisfies the equation

$$
G_{t}+v(x) \cdot \nabla G=s_{l}|\nabla G| \cdot
$$

See [26] for more details. Although solutions may develop singularities, the initial value problem with initial data $G_{0}(x) \in C^{1}$ is well-posed in the viscosity-sense. We will also consider a "viscous G equation"

$$
G_{t}+v(x) \cdot \nabla G=s_{l}|\nabla G|+\kappa \Delta G
$$

in order to determine the effect of viscosity on the large-time behavior of the front $\Gamma_{t}$.

Solutions to equations (2.2) and (2.3) admit an optimal control representation. Suppose that $u(x, t)$ satisfies $(2.2)$ for $t>0, x \in \mathbb{R}^{n}$ with initial condition $u(x, 0)=$ $u_{0}(x)$. Then $u(x, t)$ has the representation

$$
u(x, t)=\sup u_{0}(y(t))
$$

where the supremum is over all $y \in W^{1, \infty}\left([0, t] ; \mathbb{R}^{d}\right)$ satisfying $y(0)=x$ and the constraint

$$
\left|y^{\prime}(\tau)+V(y(\tau))\right| \leq s_{l}
$$


for all $\tau \in[0, t]$. This representation may be derived as in [9], Section 10.3. For the viscous equation (2.3), the control problem becomes stochastic [11, 13]. Suppose $w(x, t)$ satisfies $(2.3)$ for $t>0, x \in \mathbb{R}^{n}$ with initial condition $w(x, 0)=w_{0}(x)$, then $w(x, t)$ has the representation

$$
w(x, t)=\sup \mathbb{E}\left[u_{0}\left(Y_{t}\right)\right]
$$

where $Y_{t}$ satisfies the stochastic equation

$$
d Y_{\tau}=\left(\alpha_{\tau}-V\left(Y_{\tau}\right)\right) d \tau+\sqrt{2 \kappa} d W_{\tau}, \quad Y_{0}=x .
$$

The supremum in (2.6) is over all stochastic controls $\alpha_{\tau}$ which are progressively measureable with respect to the Brownian filtration, and satisfy $\left|\alpha_{\tau}\right| \leq s_{l}$.

3. Examples and inviscid speed bounds. In this section, we give bounds of front speeds for the inviscid G-equation with concrete examples. Consider spatial flow $v=V(x)$, and the G-equation:

$$
u_{t}+V(x) \cdot \nabla u=s_{l}\left|\nabla_{x} u\right|,
$$

with affine initial data $u(x, 0)=p \cdot x$, where $p$ is a unit vector in $\mathbb{R}^{d}$. We will assume that $p=(1,0, \ldots, 0)$. The solution is given by the control formula:

$$
u(x, t)=\sup _{\alpha} p \cdot y(t),
$$

where $y=y(t ; x)$ is the solution to the ODE with continous control $\alpha,|\alpha| \leq s_{l}$ :

$$
y^{\prime}(\tau)=-V(y(\tau))+\alpha(\tau)
$$

and initial data $y(0)=x$.

Example 1 (gradient flow). First, we analyze the one-dimensional case. Clearly, $\alpha=s_{l}$ is an admissible control. Let $p=1$. By an ODE comparison argument, $\alpha=s_{l}$ is the optimal control as it facilitates the largest velocity to the right. So the solution formula is:

$$
u(x, t)=y,
$$

where $y=y(t ; x)$ is the solution to the ODE:

$$
y^{\prime}(\tau)=-V(y(\tau))+s_{l},
$$

with initial data $y(0)=x$. Two regimes arise. If $\min -V(y)+s_{l}>0$ for all $y$, then (3.5) is solvable in closed form:

$$
\int_{x}^{y} \frac{d \eta}{-V(\eta)+s_{l}}=t
$$

To find the asymptotic front speed, send $t \rightarrow+\infty$. It follows from (3.6) that if $V$ is a stationary ergodic process, then

$$
s_{T}=\lim _{t \rightarrow \infty} u(x, t) / t=\lim _{t \rightarrow \infty} y(t) / t=1 / \mathbb{E}\left[\left(-V(\cdot)+s_{l}\right)^{-1}\right]
$$


holds almost surely. Bounding the harmonic mean in (3.7) by the arithmetic mean, we see that $s_{T} \leq s_{l}-\mathbb{E}[V]$. If also $\mathbb{E}[V]=0$, this means that $V$ has the effect of slowing the front, consistent with results for the KPP equation [21].

On the other hand, if $-V+s_{l}$ changes sign, a generic case is that $-V+s_{l}$ has discrete zeros where $-V^{\prime}$ changes sign alternately. These zeros are stable and unstable equilibria of (3.5). It is easy to see that $y(t)$ will converge to the nearest stable equilibriam from the starting point $x$, or remain motionless if $x$ happens to be one of the equilibria. In any event, $y(t)$ is uniformly bounded in $t$, implying that the asymptotic front speed $s_{T}=0$. That is, front trapping occurs at drift with large enough amplitude variations. Although this is similar to the case of bistable fronts, this is different from the KPP case where fronts slow down at large drift [21] but trapping does not happen, even though the front speed is slowed down arbitrarily close to zero. The lack of diffusion in the G-equation is the likely cause. The similarity between the G-equation front and bistable front trapping [32] suggests that the Gequation captures bistable reaction quite well.

Next, in several dimensions, let $p=(1,0, \cdots, 0)$, and let us choose a simple control $\alpha=\left(s_{l}, 0, \cdots, 0\right)$ to maximize speed along the direction $p$. Due to lack of comparison in vector ODEs, this choice only provides a lower bound of for the G-equation solution:

$$
u(x, t) \geq y_{1}(t)
$$

where:

$$
y^{\prime}=-V(y)+\left(s_{l}, 0, \cdots, 0\right)^{T}, \quad y(0)=x .
$$

If the underlying flow $y(t)$ is ergodic with invariant measure $\mu$, then as $t \rightarrow+\infty$ :

$$
y_{1}(t) / t=\left[x_{1}-\int_{0}^{t} V_{1}(y(t)) d \tau\right] / t+s_{l} \rightarrow-\mathbb{E}_{\mu}\left[V_{1}(\cdot)\right]+s_{l},
$$

almost surely in $\mu$. So, the G-equation front speed has the lower bound:

$$
s_{T} \geq-\mathbb{E}_{\mu}\left[V_{1}(\cdot)\right]+s_{l}
$$

Example 2 (shear flow). Consider a shear flow $V=\left(v\left(y_{2}\right), 0\right),\left(y_{1}, y_{2}\right) \in \mathbb{R}^{2}$, where $v\left(y_{2}\right)$ is a mean zero stationary ergodic process. Choose a control as follows:

$$
\begin{aligned}
& y_{1}^{\prime}=-v\left(y_{2}\right)+s_{l}-s_{l} \epsilon\left(\xi-x_{2}\right) e^{-\epsilon s_{l} t}, \\
& y_{2}^{\prime}=s_{l} \epsilon\left(\xi-x_{2}\right) e^{-\epsilon s_{l} t}
\end{aligned}
$$

where $\xi$ is a point so that $-v(\xi)>0$. The parameter $\epsilon \ll 1$ is chosen so that the control is admissible. Then:

$$
y_{2}(t)=x_{2}+\left(\xi-x_{2}\right)\left(1-e^{-\epsilon s_{l} t}\right) \rightarrow \xi
$$

as $t \rightarrow \infty$, and

$$
\begin{aligned}
y_{1}(t) & =x_{1}-\int_{0}^{t} v\left(y_{2}(\tau)\right) d \tau+s_{l} t+O(1) \\
& =-v(\xi) t+s_{l} t+O(1)
\end{aligned}
$$


implying that $y_{1} / t \rightarrow-v(\xi)+s_{l}$. In fact, $\xi$ may be chosen to reach the running maximum of $-v$ on the interval $\left[x_{2}-L, x_{2}+L\right]$, and so:

$$
s_{T} \geq \sup _{L} \max _{y \in[-L, L]}-v(y)+s_{l} .
$$

For a Gaussian process, $s_{T}$ diverges, implying that homogenization of the G-equation breaks down. This divergence of $s_{T}$ in unbounded shear flow is similar to the case of KPP fronts [33], and the quadratic inviscid Hamilton-Jacobi fronts [6].

If $v$ is a bounded process, however, then $s_{T}$ grows linearly like $\delta\|v\|_{\infty}$ if $v$ is scaled to $\delta v, \delta \gg 1$. This same linear scaling holds even if the shear is not aligned with $p$. For example, let

$$
V=-\delta\left(m \cos \left(-n y_{1}+m y_{2}\right), n \cos \left(-n y_{1}+m y_{2}\right)\right)=\left(-\mathcal{H}_{y_{2}}, \mathcal{H}_{y_{1}}\right),
$$

with Hamiltonian $\mathcal{H}=\delta \sin \left(-n y_{1}+m y_{2}\right),(n, m)$ are integers, $n / m$ is the rotation number. Choose the controlled system as:

$$
\begin{aligned}
& y_{1}^{\prime}=\delta m \cos \left(-n y_{1}+m y_{2}\right)+s_{l}(1-\cos t), \\
& y_{2}^{\prime}=\delta n \cos \left(-n y_{1}+m y_{2}\right),
\end{aligned}
$$

implying that:

$$
\begin{gathered}
\left(-n y_{1}+m y_{2}\right)^{\prime}=-n s_{l}(1-\cos t), \\
-n y_{1}+m y_{2}=-n x_{1}+m x_{2}-n s_{l}(t-\sin t) .
\end{gathered}
$$

So:

$$
y_{1}=x_{1}+\delta m \int_{0}^{t} \cos \left(n s_{l}\left(t^{\prime}-\sin t^{\prime}\right)+n x_{1}-m x_{2}\right) d t^{\prime}+s_{l}(t-\sin t) .
$$

For simplicity, set $x=\left(x_{1}, x_{2}\right)=0$, and $s_{l}=1 / n$. The integral in (3.16) equals:

$$
\int_{0}^{t}\left[\cos t^{\prime} \cos \left(\sin t^{\prime}\right)+\sin \left(t^{\prime}\right) \sin \left(\sin t^{\prime}\right)\right] d t^{\prime}
$$

Because the integrand is $2 \pi$ periodic, we let $t=2 \pi$. The first integrand is $(\sin \sin t)^{\prime}$, hence it suffices to consider the second integral:

$$
\int_{0}^{2 \pi} \sin \left(t^{\prime}\right) \sin \left(\sin t^{\prime}\right) d t^{\prime}
$$

As the function $G(\eta)=\eta \sin \eta$ is even, and positive over $\eta \in[0,1] \subset[0, \pi / 2]$, we conclude that the integral (3.17) is positive and

$$
s_{T} \geq \lim _{t \rightarrow \infty} y_{1} / t=\delta m(2 \pi)^{-1} \int_{0}^{2 \pi} \sin \left(t^{\prime}\right) \sin \left(\sin t^{\prime}\right) d t^{\prime}+s_{l}=O(\delta)+s_{l} .
$$

By upper bound $s_{T} \leq O(\delta)$, it follows that $s_{T}$ is enhanced linearly in large $\delta$.

Likewise, the KPP front speed obeys the linear growth law (with a precise constant rate). Recall that KPP speed $c_{*}$ in a mean zero divergence-free periodic vector field $b(x)$ is [37]:

$$
\lim _{\delta \rightarrow \infty} c_{*}(\delta, k) / \delta=\sup _{w \in D_{I}} \int_{T^{N}}(b \cdot k) w^{2}(x) d x
$$


where

$$
D_{I}=\left\{w \in H^{1}\left(T^{N}\right): b \cdot \nabla w=0,\|\nabla w\|_{2}^{2} \leq f^{\prime}(0),\|w\|_{2}=1\right\},
$$

where $k$ is the direction of front propagation, $T^{N}$ the N-dimensional unit torus. The limit in (3.18) is nonzero if $\int_{T^{N}}(b \cdot k) w_{0} d x \neq 0$ for some first integral $w_{0}$. Suppose such a $w_{0}$ exists, then $w=\left(1+\epsilon w_{0}\right) /\left\|1+\epsilon w_{0}\right\|_{2} \in D_{I}$ for $\epsilon$ small enough, and the limiting value is to leading order $\epsilon \int_{T^{N}}(b \cdot k) w_{0} d x$, which is positive if $\epsilon$ is chosen to have the sign of the integral. In our example, $k=(1,0)$, and equation $b \cdot \nabla w=0$ becomes:

$$
\left(m w_{y_{1}}+n w_{y_{2}}\right) \cos \left(-n y_{1}+m y_{2}\right)=0
$$

which has solution of the form $w_{0}=W\left(-n y_{1}+m y_{2}\right)$, for any $C^{1}$ function $W$. Then:

$$
\int_{T^{2}}(b \cdot k) w_{0} d x=\int_{T^{2}}(-\delta m) \cos \left(-n y_{1}+m y_{2}\right) W\left(-n y_{1}+m y_{2}\right) d y
$$

which is not zero if $W(\cdot)=\cos (\cdot)$. Hence, the KPP front speed also grows linearly with $\delta$ in this case.

Example 3 (cellular flow). Suppose that $V$ is the two-dimensional cellular flow

$$
V\left(y_{1}, y_{2}\right)=\delta\left(\sin \left(\pi y_{1}\right) \cos \left(\pi y_{2}\right),-\sin \left(\pi y_{1}\right) \cos \left(\pi y_{2}\right)\right)
$$

corresponding to Hamiltonian $\mathcal{H}\left(y_{1}, y_{2}\right)=(\delta / \pi) \sin \left(\pi y_{1}\right) \sin \left(\pi y_{2}\right)$. One can design a control to show the speed scaling $s_{T}(\delta)=O(\delta / \log \delta)$, for fixed $s_{l}$ and $\delta \gg 1$. The control is designed to maximize

$$
y(t) \cdot(1,0)
$$

where $y(t)=\left(y_{1}(t), y_{2}(t)\right)$ solves

$$
y^{\prime}(t)=-V(y(t))+\alpha(t)
$$

The integer lattice points are saddle points of $V$, the half integer points are centers, see Fig. 1. The controlled path $y(t)$ eventually traverses the heteroclinic orbits from one saddle point to the next, see arrowed horizontal and vertical trajectory in Fig. 1.

Suppose the initial particle position is on a separatrix. It suffices to consider the flow from saddle point $(-1,1)$ to saddle point $(0,1)$. Other motion from saddle to saddle is the same. Near the saddle points, the cell flow is very slow. Away from the saddle points, the cell flow is fast. Along this heteroclinic orbit, $-V_{1}=-\delta \sin \left(\pi y_{1}\right)>$ 0 . We set $\alpha=\left(s_{l}, 0\right)$ until the particle reaches $(0,1)$. The time required to reach $(0,1)$ is given explicitly by

$$
T_{\delta}=2 \int_{0}^{1 / 2} \frac{1}{s_{l}+\delta \sin (\pi s)} d s
$$

It is easy to see that $T_{\delta}=O\left(\delta^{-1} \log (\delta)\right)$ :

$$
\begin{aligned}
\delta T_{\delta} & =2 \int_{0}^{1 / 2} \frac{1}{s_{l} \delta^{-1}+\sin (\pi s)} d s \\
& =2 \int_{0}^{1 / 2} \frac{1}{s_{l} \delta^{-1}+\pi s} d s+2 \int_{0}^{1 / 2} \frac{\pi s-\sin (\pi s)}{\left(s_{l} \delta^{-1}+\sin (\pi s)\right)\left(s_{l} \delta^{-1}+\pi s\right)} d s .
\end{aligned}
$$




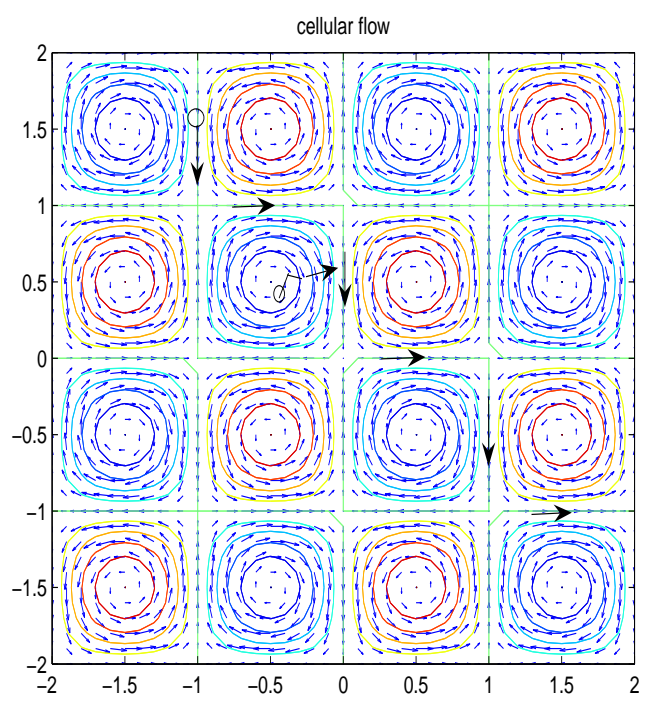

FIG. 1. Sketch of cellular flow and trajectories to maximize motion in the $x$ direction under control for initial position on or not on a separatrix.

The first term is equal to $\frac{2}{\pi} \log \left(1+\frac{\delta \pi}{s_{l}}\right)$, while the second term is bounded by a constant independent of $\delta$. Therefore,

$$
\lim _{\delta \rightarrow \infty} \frac{\delta T_{\delta}}{\log (\delta)}=\frac{2}{\pi}
$$

Now, we use the same strategy to travel to other saddle points as shown in Fig. 1. The time it takes to travel from one saddle point to the next is equal to $T_{\delta}$. At time $t$, the $y_{1}$-coordinate of $y(t)$ will be bounded from below by $y_{1}(t) \geq \frac{t}{2 T_{\delta}}-1$, implying that

$$
s_{T} \geq \frac{1}{2 T_{\delta}}=O(\delta / \log (\delta))
$$

If the initial position $x_{1}$ is away from the separatrices, it takes finite amount of time for the control vector to move the particle to a nearby separatrix, then follow the above heteroclinic motion. For example, suppose the particle is turning around inside the initial cell with corner points $(-1,0),(-1,1),(0,0),(0,1)$ in Fig. 1. If $V\left(y_{1}, y_{2}\right)=$ $\nabla^{\perp} \mathcal{H}\left(y_{1}, y_{2}\right)$ with $\mathcal{H}=0$ defining the separatrices, then we choose the control

$$
\alpha(s)=-s_{l} \frac{\nabla \mathcal{H}(y(s))}{|\nabla \mathcal{H}(y(s))|} \cdot \operatorname{sign}(\mathcal{H}(y(s)))
$$

until the particle reaches a separatrix. In this way, $\mathcal{H}(y(s))$ is either monotone increasing or decreasing in $s$ until $y(s)$ reaches the separatrix, which occurs in finite time independent of $\delta$. At that point, we may change the strategy to the one described above.

An upper bound on the speed follows in a similar manner. If $y_{1} \in[0,1]$, we have $\left|y_{1}^{\prime}(t)\right| \leq\left|V_{1}(y(t))\right|+s_{l} \leq \delta \sin \left(\pi y_{1}(t)\right)+s_{l}$, for any choice of the control. 
Consequently, the time required to traverse the strip $y_{1} \in[0,1]$, is bounded below by $T_{\delta}$. By periodicity, an amount of time at least $T_{\delta}$ is required to traverse each strip of the form $y_{1} \in[k, k+1]$, with $k \in \mathbb{Z}$. Therefore, for any $t>0, y_{1}(t) \leq \frac{t}{T_{\delta}}+1$ must hold, which implies that $s_{T} \leq \frac{1}{T_{\delta}}$. Combining this with (3.25), we conclude that

$$
\frac{\pi}{4} \leq \liminf _{\delta \rightarrow \infty} \frac{\log (\delta) s_{T}(\delta)}{\delta} \leq \limsup _{\delta \rightarrow \infty} \frac{\log (\delta) s_{T}(\delta)}{\delta} \leq \frac{\pi}{2} .
$$

A similar analysis when the initial starting position $x$ falls on a heteroclinic orbit is given in [29]. See also formal arguments based on a reduced front equation in [1].

4. The viscous G-equation. Example 3 of the last section shows that the asymptotic front speed for the inviscid G-equation scales as $O(\delta / \log \delta)$ in large amplitude $O(\delta)$ cell flows, very different from the $O\left(\delta^{1 / 4}\right)$ scaling of KPP front speeds $[2,22]$. This motivates us to study the viscous G-equation:

$$
u_{t}+\delta V(x) \cdot \nabla u=s_{l}|\nabla u|+\kappa \Delta u,
$$

where $\left(s_{l}, \kappa\right) \ll \delta$, with Lipschitz continuous initial data. Recall that the KPP front speeds come from an analogous quadratic Hamilton-Jacobi equation, or (4.1) with $|\nabla u|^{2}$ replacing $|\nabla u|$. Here $V(x)$ is a mean zero, divergence-free, periodic cellular flow.

The traveling front solution of (4.1) is:

$$
u=p \cdot x+H(p) t+w(x),
$$

where $p$ is a vector in $\mathbb{R}^{d}$, and $w(x)$ is periodic. Substituting (4.2) in (4.1), we find the equation for $(H, w(x))$ :

$$
H+\delta V(x) \cdot\left(p+\nabla_{x} w\right)=s_{l}\left|p+\nabla_{x} w\right|+\kappa \Delta_{x} w, \quad x \in \mathbb{T},
$$

where $\mathbb{T}$ is the periodic cell, equal to $[0,2] \times[0,2]$ for two dimensional cell flows. This is the cell-problem of homogenization of (4.1).

This is a nonlinear eigenvalue problem for which the unknown constant $H(p)$ is given by

$$
H(p)=s_{l}\langle|p+\nabla w|\rangle,
$$

where the bracket is the periodic average over $\mathbb{T}$. By Jensen's inequality, we have from (4.4) the lower bound $H(p) \geq s_{l}|p|$. Therefore, the speed of the propagating front is bounded below by

$$
c^{*}=H(p) /|p| \geq s_{l},
$$

so the flow can only enhance the front speed.

Now let us address the speed scaling question: if the dimension is $d=2, V$ is cell flow, how does $c^{*}$ scale with $\delta \gg 1$ ? Is it related to $O\left(\delta^{1 / 4}\right)$, as in the KPP case? Our main result is the following:

Theorem 4.1. Let $V$ be a cellular flow in $\mathbb{R}^{2}$. Let $p=(\lambda, 0)$ and $\lambda \neq 0$, in equation (4.3). If the ratio $s_{l} / \kappa$ is sufficiently small, then there is a constant $C=C(\lambda, p)$ such that the front speed $c^{*}$ satisfies

$$
s_{l} \leq c^{*} \leq s_{l} C(1+\sqrt{\log (\delta / \kappa)}),
$$


for all $\delta>\kappa$.

Proof. It will be convenient to write the cell problem (4.3) as

$$
H^{*}+A V(x) \cdot\left(p+\nabla_{x} w\right)=R\left|p+\nabla_{x} w\right|+\Delta_{x} w, \quad x \in \mathbb{T},
$$

where $A=\delta / \kappa$ and $R=s_{l} / \kappa$ and $H^{*}=H / \kappa$. Observe that the front speed is $c^{*}=H^{*} \kappa /|p|$.

As in [22], we decompose $(p=(\lambda, 0))$ :

$$
p \cdot x+w=\lambda x_{1}+w=T+S \equiv \zeta,
$$

where $T$ has zero average over $[-1,1]^{2}$ and solves the linear inhomogeneous problem:

$$
\begin{aligned}
& \Delta T-A V(x) \cdot \nabla T=0, \\
& T\left(x_{1}+2, x_{2}\right)=T\left(x_{1}, x_{2}\right)+2 \lambda, T\left(x_{1}, x_{2}+2\right)=T\left(x_{1}, x_{2}\right),
\end{aligned}
$$

and $S$, a mean zero periodic function, solves the nonlinear problem:

$$
\Delta S-A V(x) \cdot \nabla S=H^{*}-R|\nabla(T+S)| .
$$

The linear problem (4.8) is well-studied and the $L^{2}$ gradient estimate is $[10,12$, 22, 23]:

$$
C_{1} A^{1 / 2} \lambda^{2} \leq \int_{\mathbb{T}}|\nabla T|^{2} d x \leq C_{2} A^{1 / 2} \lambda^{2}
$$

for positive constants $C_{1}, C_{2}$ independent of $A$.

Lemma 4.1. There is a positive constant $C$ independent of $A$ such that

$$
\|\nabla T\|_{L^{1}(\mathbb{T})} \leq C(1+\sqrt{\log A})
$$

holds for all $A \geq 1$.

Proof of Lemma. Let $\Omega(a, h)=\{x \in \mathbb{T}|a<| \mathcal{H}(x) \mid<a+h\}$, for any positive $a$ and $h$. Here $\mathcal{H}$ is the Hamiltonian with $\nabla^{\perp} \mathcal{H}=V\left(\right.$ e.g. $\left.\mathcal{H}\left(y_{1}, y_{2}\right)=\sin \left(\pi y_{1}\right) \sin \left(\pi y_{2}\right)\right)$. Letting $\epsilon=1 / A$, we have:

$$
|\Omega(a, \sqrt{\epsilon})| \leq O(\sqrt{\epsilon}(1+|\log (\epsilon)|)) .
$$

By Theorem 4.2 of [23], for $N \geq 1$ :

$$
\|\nabla T\|_{L^{2}(\{x \in \mathbb{T}:|H| \geq N \sqrt{\epsilon}\})}^{2} \leq \frac{C}{\sqrt{\epsilon} N^{4}} .
$$

It follows that

$$
\begin{aligned}
\|\nabla T\|_{L^{1}(\mathbb{T})}= & \|\nabla T\|_{L^{1}(\{x \in \mathbb{T}:|H| \leq \sqrt{\epsilon}\})}+\sum_{N=1}^{\infty}\|\nabla T\|_{L^{1}(\Omega(N \sqrt{\epsilon}, \sqrt{\epsilon}))} \\
\leq & \|\nabla T\|_{L^{2}(\mathbb{T})} \sqrt{|\{x \in \mathbb{T}:|H| \leq \epsilon\}|} \\
& +\sum_{N=1}^{\infty}\|\nabla T\|_{L^{2}(\Omega(N \sqrt{\epsilon}, \sqrt{\epsilon}))} \sqrt{|\Omega(N \sqrt{\epsilon}, \sqrt{\epsilon})|} \\
\leq & C \sqrt{\sqrt{\epsilon}(1+|\log (\epsilon)|)}\left(1+\sum_{N=1}^{\infty} N^{-2}\right) \epsilon^{-1 / 4} \\
\leq & C \sqrt{1+|\log (\epsilon)|} \leq C(1+\sqrt{\log A}) .
\end{aligned}
$$


REMARK 4.1. The $L^{1}$ gradient estimate in Lemma 4.1 can also be proved based on Theorem 3.2 of [23] with the upper bound there $(C / H)\left(\epsilon / H^{2}\right)^{3 / 8}$ corrected to $(C / H)\left(\epsilon / H^{2}\right)^{3 / 2}$. The exponent $3 / 8$ results from a miscount of exponent in the last three lines of the proof of Theorem 3.2 of [23].

The function $\zeta=w+p \cdot x$ increases by $\lambda$ across the cell in $x_{1}$. It follows from (4.4) that:

$$
\begin{aligned}
H^{*}(p) & =R \int_{\mathbb{T}}|\nabla \zeta| d x /|\mathbb{T}| \geq R \int_{\mathbb{T}}\left|\zeta_{x}\right| d x /|\mathbb{T}| \\
& \geq R \int_{\mathbb{T}} \zeta_{x_{1}} d x /|\mathbb{T}|=R \lambda
\end{aligned}
$$

Since $\zeta$ satisfies $\Delta \zeta-A V \cdot \nabla \zeta+|\nabla \zeta|=H^{*}>0$, Proposition 4 of [22] may be extended to show that $\zeta$ satisfies the upper bound:

$$
\zeta \leq C\left[\lambda+\|\nabla \zeta\|_{1}\right]
$$

in the cell $\mathbb{T}$. This follows in a straightforward manner, from the maximum principle and Poincaré inequality (for $L^{1}$ norm rather than $L^{2}$ ), as in [22]. This bound implies that

$$
\zeta \leq C\left[\lambda+H^{*} / R\right] \leq C H^{*} / R,
$$

for a positive universal constant $C$. The type of inequality (4.14) also applies to $T$ and gives:

$$
T \leq C\left[\lambda+\|\nabla T\|_{1}\right] \leq C(\lambda+1+\sqrt{\log A})
$$

for all $A>1$. Inequality (4.16) improves Lemma 1 of [22] where the upper bound is $O\left(\lambda A^{1 / 4}\right)$. Let us mention that a special $L^{\infty}$ estimate is:

$$
\|T\|_{\infty} \leq \lambda
$$

if $H$ is odd in $x_{1}[10]$.

It follows from (4.16) and (4.15) that

$$
S \leq C\left(1+\sqrt{\log A}+H^{*} / R\right) .
$$

By (4.4)-(4.7):

$$
|\mathbb{T}| H^{*}=R\|\nabla(T+S)\|_{1}
$$

and so:

$$
\left\|\mathbb{T}\left|H^{*}-R\|\nabla T\|_{1}\right| \leq R\right\| \nabla S\left\|_{1} \leq 2 R\right\| \nabla S \|_{2} .
$$

Multiplying $S$ to (4.9), integrating over $\mathbb{T}$ and applying (4.18) gives:

$$
\begin{aligned}
\|\nabla S\|_{2}^{2} & =R \int_{\mathbb{T}} S|\nabla(T+S)| d x \\
& \leq R C\left(1+\sqrt{\log A}+H^{*} / R\right) \int_{\mathbb{T}}|\nabla \zeta| d x \\
& =C\left(1+\sqrt{\log A}+H^{*} / R\right) H^{*}
\end{aligned}
$$


It follows from (4.19) that:

$$
\begin{aligned}
& |\mathbb{T}| H^{*} \leq 2 R\|\nabla S\|_{2}+R\|\nabla T\|_{1} \\
& \leq 2 R \sqrt{C\left(1+\sqrt{\log A}+H^{*} / R\right) H^{*}}+R C(1+\sqrt{\log A}) .
\end{aligned}
$$

Suppose $H^{*}>R(1+\sqrt{\log A})$. Then (4.21) implies

$$
|\mathbb{T}| H^{*} \leq R \sqrt{2 C H^{*} H^{*} / R}+R C(1+\sqrt{\log A}) .
$$

So, if $\sqrt{R}=\sqrt{s_{l} / \kappa}$ is sufficiently small (independently of $A$ ), $H^{*} \leq R C(1+\sqrt{\log A}$ ). Therefore, there is a constant $C$ independent of $A$ such that

$$
H^{*} \leq R C(1+\sqrt{\log A}),
$$

holds for all $A>1$. Consequently,

$$
c^{*}=H /|p|=H^{*} \kappa /|p| \leq s_{l} C(1+\sqrt{\log (\delta / \kappa)})
$$

holds for $\delta>\kappa$. प

Consider the quadratically nonlinear HJ where $\left|p+\nabla_{x} w\right|^{2}$ replaces $\left|p+\nabla_{x} w\right|$ in (4.6). Then the lower bound of $H^{*}$ is (two lines under (3.43) in [22]):

$$
\left(H^{*}\right)^{1 / 2}+O\left(H^{*}\right)+O\left(\left(H^{*}\right)^{3 / 4}\right) \geq O\left(\lambda A^{1 / 4}\right),
$$

implying:

$$
H^{*} \geq O\left(\lambda A^{1 / 4}\right)
$$

or:

$$
c^{*} \geq C(\lambda, \kappa) \delta^{1 / 4}
$$

at fixed $\kappa$ and $\lambda$. In other words, the front speed for the quadratically nonlinear HJ equation grows at least as $O\left(\delta^{1 / 4}\right)$ at large $\delta$. The order of the nonlinearity makes a significant difference in the asymptotic behavior of the front. In contrast, nonlinearities in reaction-diffusion equations do not change the scaling behavior of speed growth in cellular flows so drastically. For ignition nonlinearity and so by comparison for all nonnegative reactive nonlinearities, the front speed in cellular flows scales as $O\left(\delta^{1 / 4}\right)$ at large $\delta$, [38].

For the inviscid G-equation, an $O(\delta / \log \delta)$ lower bound on the front speed followed from a choice of control which steers the trajectory $y(t)$ along the separatrices, taking advantage of the strong flow away from the stagnation points. We may also consider the control representation for the viscous G-equation (4.1):

$$
u(t, x)=\sup _{\alpha} \mathbb{E}\left[p \cdot X_{t}\right],
$$

where

$$
d X_{t}=\left(\alpha_{t}-\delta V\left(X_{t}\right)\right) d t+\sqrt{2 \kappa} d W_{t},
$$

and $\alpha_{t}$ is a control, adapted to the Brownian filtration and satisfying $\left|\alpha_{t}\right| \leq s_{l}$. The supremum is over all such controls. The reduction of enhancement due to viscosity 
$\kappa>0$ in the above theorem can be explained as follows. The presence of strong enough Brownian perturbation kicks the particle from moving near the separatrices (Fig. 1) towards the center of the cell where temporary trapping occurs. If the particle is close to a separatrix, then the flow is $O(\delta)$ in that region, so it has time of order $O\left(\delta^{-1}\right)$ in which to hop to the adjecent cell before being transported around to the other side of the cell. In this short time interval, the Brownian path makes typical excursions of the size $O\left(\delta^{-1 / 2}\right)$, much larger than $O\left(\delta^{-1}\right)$ which is the amount the control is able to influence the trajectory over the same interval. In order to steer the particle across a separatrix, the particle must first be within a distance $O\left(\delta^{-1}\right)$ of the separatrix. In this range, the random perturbations may easily move the particle away from the separatrix (with probability close to $1 / 2$ ). In the worst case, the particle is transported quickly around the cell to the opposite separatrix, where stochastic pertubations may then kick the particle across the separatrix, in the opposite direction (in the direction $-p)$. Hence the transition from one cell to the next is dominated by stochastic effects which suggests large-scale diffusive behavior of the trajectories, rather than ballistic behavior in the direction of $p$. Random perturbations in the regime of $s_{l} \ll \kappa \ll \delta$ weaken the effect of control velocity $\alpha_{t}$ which is the mechanism of the much faster speed scaling $O(\delta / \log \delta)$ in the inviscid G-equation. It is interesting to find out what happens in the opposite regime $0<\kappa \ll s_{l} \ll \delta$.

5. Conclusions. Front speeds for the inviscid G-equation in gradient flows and shear flows behave qualitatively similar to KPP speeds. However, front speeds for the G-equation in cellular flows are very different. The inviscid G-front speed grows at a nearly linear rate at large cellular flow amplitude, while the viscous G-equation front speed has much slower growth. It remains to study the interesting regime of slightly viscous G-equation where the viscosity coefficient is much smaller than the laminar speed. It also would be interesting to determine whether there is an $O(\sqrt{\log (\delta / \kappa}))$ lower bound on the front speed for viscous G-equation in cellular flows. The central issue is whether the $L^{1}$ gradient estimate of Lemma 4.1 is optimal.

\section{REFERENCES}

[1] M. Abel, M. Cencini, D. Vergni And A. Vulpiani, Front speed enhancement in cellular flows, Chaos, 12:2 (2002), pp. 481-488.

[2] B. Audoly, H. Berestycki and Y. Pomeau, Réaction-diffusion en écoulement stationnaire rapide, C. R. Acad. Sci, Paris 328 II (2000), pp. 255-262.

[3] G. Barles and P.E. Souganidis, A New Approach to Front Propagation Problems: Theory and Applications, Arch Rational Mech. Analysis, 141 (1998), pp. 237-296.

[4] P. Constantin, A. Kiselev, A. Oberman, and L. Ryzhik, Bulk burning rate in passivereactive diffusion, Arch. Ration. Mech. Anal., 154 (2000), pp. 53-91.

[5] B. Denet, Possible role of temporal correlations in the bending of turbulent flame velocity, Combust Theory Modelling, 3 (1999), pp. 585-589.

[6] W. E, J. Wehr, AND J. XIN, Breakdown of homogenization for the random Hamilton-Jacobi equations, Comm. Math Sci, 6:1 (2008), pp. 189-197.

[7] P. Embid, A. Majda, And P. Souganidis, Comparison of turbulent flame speeds from complete averaging and the G-equation, Phys. Fluids, 7 (1995), pp. 2052-2060.

[8] L. C. Evans, Periodic homogenization of certain fully nonlinear partial differential equations, Proc. Royal Soc. Edingburgh, Section A, 120 (1992), pp. 245-265.

[9] L. C. Evans, Partial Differential Equations, Graduate Studies in Mathematics, AMS, Providence, 1998.

[10] A. Fannjiang and G. Papanicolaou, Convection enhanced diffusion for periodic flows, SIAM J. Applied Math, 54 (1994), pp. 333-408.

[11] W. Fleming And H. Soner, Controlled Markov Processes and Viscosity Solutions, Applications of Mathematics 25, Springer-Verlag, 1993. 
[12] S. Heinze, Diffusion-Advection in Cellular Flows with Large Péclet Numbers, Arch. Rational Mech Anal., 168 (2003), pp. 329-342.

[13] E. Kosygina, F. Rezakhanlou, and S. Varadhan, Stochastic homogenization of HamiltonJacobi-Bellman equations, Comm. Pure Appl. Math., 59 (2006), pp. 1489-1521.

[14] E. Kosygina AND S. VARAdhan, Homogenization of Hamilton-Jacobi-Bellman equations with respect to time-space shifts in a stationary ergodic medium, Comm. Pure Appl. Math, 61 (2008), pp. 816-847.

[15] P.-L. Lions, G. Papanicolaou and S. Varadhan, Homogenization of Hamilton-Jacobi equations, unpublished preprint, circa 1986.

[16] P.-L. Lions and P. Souganidis, Homogenization of viscous Hamilton-Jacobi equations in stationary ergodic media, Comm. Partial Diff. Eqn., 30 (2005), pp. 335-375.

[17] P.-L. Lions and P. Souganidis, Homogenization of degenerate second order PDE in periodic and almost periodic environments and applications, Ann Inst. H. Poincaré, Analyse Non Lineaire, 22 (2005), pp. 667-677.

[18] A. J. MAJdA AND P. E. Souganidis, Large scale front dynamics for turbulent reaction-diffusion equations with separated velocity scales, Nonlinearity, 7 (1994), pp. 1-30.

[19] G. Markstein, Nonsteady Flame Propagation, Pergamon Press, Oxford, 1964.

[20] J. Nolen And J. XIN, Reaction-diffusion front speeds in spatially-temporally periodic shear flows, SIAM J. Multiscale Modeling and Simulation, 1 (2003), pp. 554-570.

[21] J. Nolen And J. XIN, KPP Fronts in a One Dimensional Random Drift, Discrete and Continuous Dynamical Systems-B, 11 (2009), pp. 421-442.

[22] A. Novikov And L. Ryzhik, Boundary layers and KPP fronts in a cellular flow, Arch. Rational Mech. Analysis, 184 (2007), pp. 23-48.

[23] A. Novikov, G. Papanicolaou, And L. Ryzhik, Boundary layers for cellular flows at high Péclet numbers, Comm. Pure Appl. Math, 58 (2005).

[24] S. Osher And R. Fedkiw, Level Set Methods and Dynamic Implicit Surfaces, Applied Math Sci, 153, Springer, New York, 2003.

[25] N. Peters, A spectral closure for premixed turbulent combustion in the flamelet regime, J. Fluid Mech., 242 (1992), pp. 611-629.

[26] N. Peters, Turbulent Combustion, Cambridge Univ Press, 2000.

[27] F. Rezakhanlou and J. Tarver, Homogenization for Stochastic Hamilton-Jacobi Equations, Arch. Rational Mech. Anal, 151 (2000), pp. 277-309.

[28] P. Ronney, B. Haslam, and N. Rhys, Front propogation rates in randomly stirred media, Phys. Rev. Lett., 74 (1995), pp. 3804-3807.

[29] A. Oberman, Ph.D Thesis, University of Chicago, 2001.

[30] G. Sivashinsky, Cascade-renormalization theory of turbulent flame speed, Combust. Sci. Tech., 62 (1988), pp. 77-96.

[31] P. Souganidis, Stochastic homogenization of Hamilton-Jacobi equations and some applications, Asymptotic Analysis, 20 (1999), pp. 1-11.

[32] J. XIN, Front Propagation in Heterogeneous Media, SIAM Review, 42 (2000), pp. 161-230.

[33] J. Xin, KPP Front Speeds in Random Shears and the Parabolic Anderson Problem, Methods and Applications of Analysis, 10 (2003), pp. 191-198.

[34] J. XIN, An Introduction to Fronts in Random Media, Surveys and Tutorials in the Applied Math Sciences, Vol. 5, Springer, 2009.

[35] V. Yакнот, Propagation velocity of premixed turbulent flames, Combust. Sci. Tech, 60 (1988), pp. 191-241.

[36] A. ZLAToš, Pulsating front speed-up and quenching of reaction by fast advection, Nonlinearity, 20 (2007), pp. 2907-2921.

[37] A. ZLAToš, Sharp asymptotics for KPP pulsating front speed-up and diffusion enhancement by flows, to appear in Arch. Ration. Mech Analysis.

[38] A. Zlatoš, Reaction-Diffusion Front Speed Enhancement by Flows, preprint, 2009. 